Original Paper http://ajol.info/index.php/ijbcs http://indexmedicus.afro.who.int

\title{
Rôle des Collectivités Territoriales Décentralisées dans la pérennisation des services d'eau et assainissement dans les zones non concédées : cas de la Commune de Dschang
}

\author{
Emile TEMGOUA $^{1^{*}}$, Vivien MELI MELI ${ }^{2}$, Modeste MEKUI ${ }^{3}$ et \\ Barthélémy NDONGSON ${ }^{3}$
}

\footnotetext{
${ }^{1}$ Faculté d'Agronomie et des Sciences Agricoles, Université de Dschang, BP 222 Dschang, Cameroun.

${ }^{2}$ Université de Dschang, Faculté des Lettres et des Sciences Humaines, Cameroun.

${ }^{3}$ Agence municipale de l'eau et énergie, Services Techniques, Commune de Dschang, BP 169 Dschang, Cameroun.

*Auteur correspondant; E-mail: etemgoual@yahoo.com
}

\section{REMERCIEMENTS}

Ce travail, réalisé par le Groupe d'Initiative Economique de l'Université de Dschang, a bénéficié du soutien financier de la Commune de Dschang et de son partenaire au développement, la ville de Nantes.

\section{RESUME}

La décentralisation actuelle dans les pays africains délègue aux Collectivités Territoriales Décentralisées (CTD) le rôle de l'approvisionnement en eau potable et assainissement (AEPA) aux populations. Elle fait des communes de nouveaux acteurs du secteur Eau-Assainissement que les associations d'opérateurs comme l'Association Africaine de l'Eau devront désormais considérer. Avec peu de ressources financières et techniques, de nombreux ouvrages d'AEPA, entrepris par les CTD, aussitôt construits sont non fonctionnels. Cette étude avait pour objectif d'auditer les services d'AEPA dans la Commune de Dschang et de proposer un modèle de pérennisation des ouvrages. La collecte des données a consisté en l'administration d'un questionnaire semi-structuré et aux observations de terrain. 511 personnes ont été enquêtées, impliquant l'ensemble des acteurs et des cibles engagés dans la conception, la mise en œuvre et l'exploitation des ouvrages d'AEPA dans la Commune. Les résultats indiquent que la mise en place d'un projet d'AEPA doit prendre en compte des contextes socioculturels pour que les projets remplissent leurs objectifs d'amélioration de la santé et des conditions de vie des populations. La mise en place d'une agence municipale dédiée à la régie de l'eau et de l'assainissement encadrerait indubitablement la pérennisation des ouvrages. Ce modèle exploité par la Commune de Dschang a favorisé la création d'un établissement public communal, et la construction des miniréseaux d'eau avec désormais un fonctionnement pérenne.

(C) 2019 International Formulae Group. All rights reserved

Mot clés : Eau, assainissement, service alternatif, régie communale AEPA, Commune de Dschang, Cameroun.

\section{Role of Decentralized Territorial Collectivities in the Sustainability of Water and Sanitation Services in non-committed Areas: Case of the Dschang Municipality}

\begin{abstract}
The current decentralization process in African countries delegates to the Decentralized Territorial Collectivities (CTD) the role of providing drinking water and sanitation (WASH) to the populations. It makes
\end{abstract}


municipalities new actors in the water-sanitation sector that operators associations such as the African Water Association (AfWA) will now have to consider. With few financial and technical resources, many WASH utilities, undertaken by the CTDs, immediately built are non-functional. The purpose of this study was to audit the WASH services in the Dschang municipality, and to propose a model for the sustainability of decentralized WASH utilities. Data collection was based on a semi-structured questionnaire administrated and field observations. 511 people were surveyed, involving all actors and targets involved in the design, implementation and operation of WASH utilities in the Council. The results indicate that the implementation of a WASH project must take into account socio-cultural contexts for the projects to fulfill their objectives of improving the health and living conditions of the populations. The establishment of a municipal agency dedicated to the management of water and sanitation would undoubtedly support the sustainability of the structures. This model operated by the Dschang Municipality has favored the creation of a WASH' council board, and the construction of mini-water networks with now a sustainable operation.

(C) 2019 International Formulae Group. All rights reserved

Keywords: Water, sanitation, alternative service, WASH council board, Dschang municipality, Cameroon.

\section{INTRODUCTION}

L'accès à l'eau potable et à l'assainissement sont deux conditions essentielles à l'atteinte des objectifs du millénaire et à la réduction de la pauvreté. Accélérer l'accès à l'assainissement et l'eau en Afrique face aux changements climatiques représente un enjeu de taille qui a été reconnu par l'ensemble des participants au $19^{\mathrm{e}}$ congrès de l'Association Africaine de l'Eau (AAE). Lors de ce congrès tenu à Bamako en février 2018, les discussions ont attiré l'attention sur la nécessité de conduire des actions en direction de l'Objectif 6 des ODD qui exige de "garantir l'accès de tous à l'eau et à l'assainissement et assurer une gestion durable des ressources en eau". Au Cameroun, le taux d'accès à l'eau potable atteint à peine $61 \%$ en 2014, et l'assainissement 40\% (INS, 2016). L'approvisionnement en eau potable se présente sous différentes formes (réseaux, puits, sources, rivières et fleuves, eaux de pluies) et varie en fonction des différentes zones d'habitation (Tanawa et al., 2002).

Aujourd'hui, la principale difficulté à laquelle les pays d'Afrique subsaharienne sont confrontés n'est pas tant cet accès à l'eau mais plus précisément l'accès à l'eau potable. En effet le problème se pose en terme de qualité des ressources en eau et c'est sur ce point que toutes les attentions se tournent, au Cameroun comme ailleurs (Temgoua, 2011;
Agassounon Djikpo Tchibozo et al., 2012; Dovonou et al., 2017; Djegbe et al., 2018). Ces pays disposent d'un nombre très élevé de forages, notamment en milieux ruraux (environ 70000 au Burkina Faso, plus de 700 forages pour la seule ville de Dschang au Cameroun en 2009 (Temgoua et al., 2009)). Le risque reste important car les services techniques ne possèdent pas une base réelle sur la connaissance du milieu souterrain et la gestion des ressources en eau. En effet, l'eau peut être le véhicule d'un très grand nombre d'agents pathogènes rejetés dans le milieu extérieur par les matières fécales humaines ou animales et peut être ainsi à l'origine de nombreuses maladies hydriques (Temgoua, 2011 ; Agassounon Djikpo Tchibozo et al., 2012 ; Dovonou et al., 2017 ; Djegbe et al., 2018). En 1996, l'OMS chiffrait à 4 milliards les nombres d'épisodes diarrhéiques survenus dans le monde, responsables de la mort de 3,1 millions de personnes dont pour la grande majorité des enfants de moins de cinq ans. A la lumière de ces chiffres, on se rend compte de l'importance du problème de l'accès à l'eau potable et $\mathrm{du}$ besoin capital de rechercher des solutions permettant d'améliorer la situation dans ce secteur.

La décentralisation actuelle dans les pays africains délègue aux Collectivités Territoriales Décentralisées (CTD) le rôle d'offrir de l'eau potable et les services 
d'assainissement aux populations. Les lois camerounaises du 22 Juillet 2004 et du 26 février 2010 vont dans ce sens. Elles font donc des communes de nouveaux acteurs du secteur Eau-Assainissement. Dans ce contexte, des modes de "gestion communautaire" (ce qualificatif ambigu pouvant aussi bien renvoyer à l'autogestion qu'à une gestion confiée à une "communauté") ont été préconisés en tant qu'alternatives, sans atteindre les objectifs affichés (Baron et Bonnassieux, 2011). D'où la question suivante posée par Olivier de Sardan et Elhadji Dagobi (2001) : la gestion communautaire sert-elle l'intérêt public ?

Les ressources financières transférées tardent à suivre et du coup, chaque localité développe ses propres stratégies pour assurer les services transférés. De nombreux ouvrages aussitôt construits sont non fonctionnels (Tanawa et al., 2002 ; Lobina et Hall, 2003 ; Temgoua et al., 2009). Cette étude avait pour objectif d'auditer ces cas d'échecs dans la Commune de Dschang et de proposer un modèle de pérennisation des ouvrages chèrement acquis. Il s'agit pour l'essentiel de :

$>$ Comprendre les représentations collectives liées à l'eau en général et aux projets d'eau et assainissement mis en œuvre dans la Commune de Dschang en particulier ;

> Présenter les acquis des solutions endogènes pour l'AEPA durable de la commune.

\section{MATERIEL ET METHODES}

\section{Présentation de la Commune de Dschang}

Dschang est une ville de Montagne (1400 m altitude) située à l'Ouest du Cameroun (Figure 1), une ville universitaire depuis 1993 (environ 35.000 étudiants en 2018). La population de la Commune de Dschang (zone urbaine et rurale) est estimée à 220.000 habitants. En effet, depuis l'installation dans la ville d'une université en 1993, la ville est sujette à un accroissement mal contrôlé de la population. La zone à habitat spontané est constituée des quartiers du marché B, Mingmeto, Ngui, Haoussa, New-town Foto et une partie du quartier Mingou. C'est un habitat de type traditionnel ou rural implanté de manière désordonné sans aucune infrastructure dont la qualité peut être améliorée par des opérations de restructuration: Ouverture des voies, installation des réseaux divers, régularisation de la situation foncière... Chaque habitant doit, dans la mesure du possible, construire des installations d'approvisionnement en eau (puits notamment) et d'assainissement (latrine). A cela, il faudra ajouter le fait que la Commune de Dschang collecte moins de $30 \%$ des déchets de la ville. Le reste des ordures est évacué dans les caniveaux, les champs et les espaces vides (Temgoua et al., 2009). En ce qui concerne les latrines à fond perdu, qui côtoient les points d'eau, elles sont le plus souvent creusées jusqu'à la nappe phréatique et accueillent les défections des habitants. Malheureusement, le système de vidange de la ville n'est plus en état de marche depuis quelques années déjà, obligeant les habitants à couvrir les anciens trous et à en creuser des nouveaux. Les anciennes fosses restent ainsi enfouies, devenant une source de pollution durable pour la nappe phréatique. Avec les moyens limités de la commune, la situation d'AEPA est telle qu'on note l'absence des latrines dans plusieurs écoles et collèges. La commune n'est pas le maître d'ouvrage en matière de distribution de l'eau dans la ville, mais le décret de 2010 sur la décentralisation du service de l'eau est une opportunité à saisir.

\section{Méthodes}

La collecte des données quantitatives a consisté en l'élaboration d'un questionnaire approprié, semi-structuré, sur le terrain par des enquêteurs, étudiants des sciences sociales, par ailleurs formés pour l'occasion. 511 personnes ont été enquêtées, dans une perspective participative, impliquant l'ensemble des acteurs et des cibles engagés 


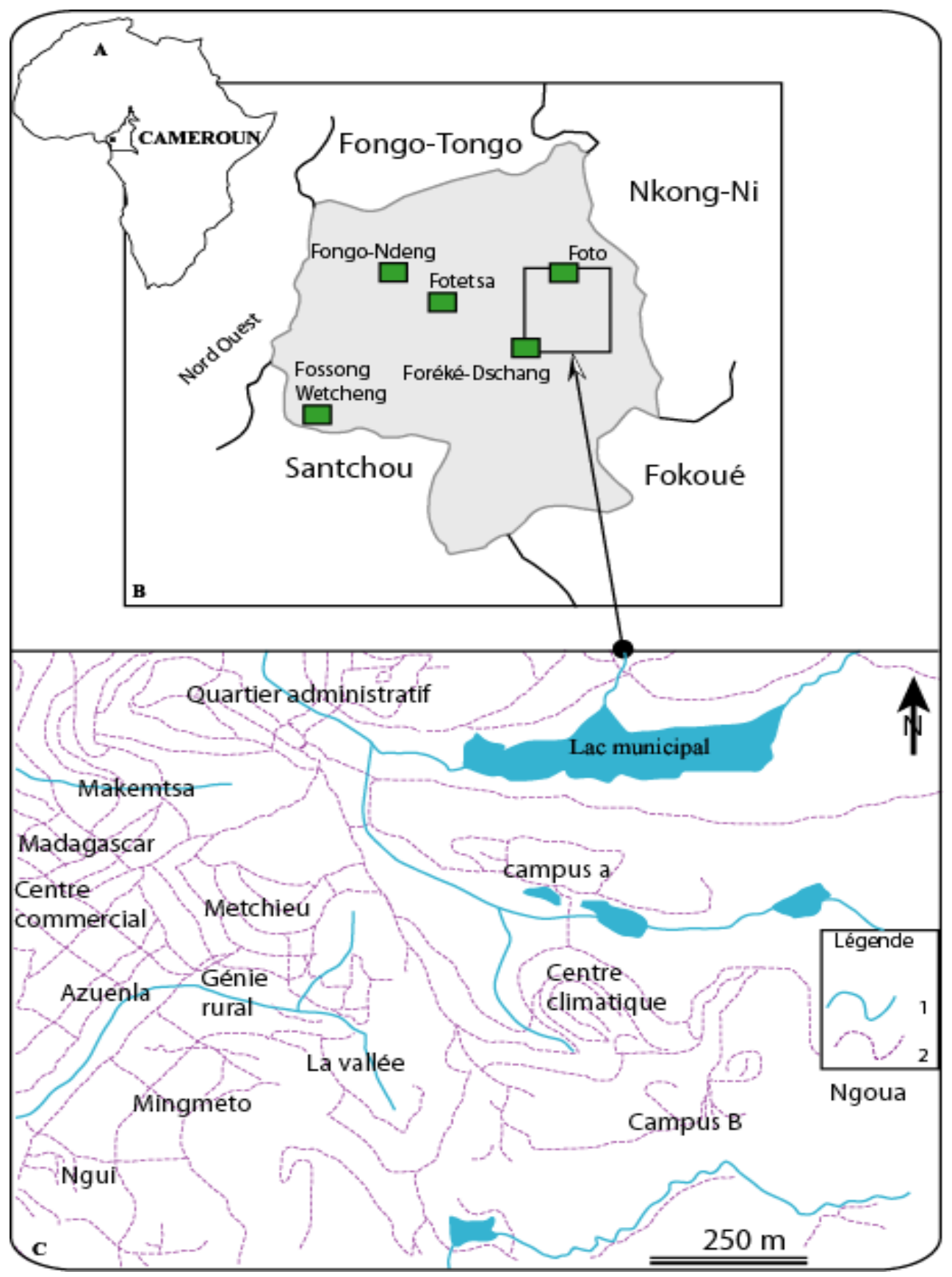

Figure 1: Carte de localisation de la Commune de Dschang, en Afrique et au Cameroun (A), dans le département de la Menoua (B) et présentation de la zone urbaine $(\mathrm{C}$, avec $1=$ cours d'eau, $2=$ voies de communication) 
dans la conception, la mise en œuvre et l'exploitation/l'utilisation des ouvrages d'eau et d'assainissement dans la Commune de Dschang. Une triangulation a été faite des données quantitatives et qualitatives.

Les données qualitatives sont issues des observations directes sur les différents sites des projets et par des échanges avec les bénéficiaires et les gestionnaires des différents projets mis en œuvre. Les procédures de création et le fonctionnement de l'agence municipale en charge de l'eau au sein de la Commune de Dschang ont été auditées à travers les observations directes de terrain et en consultant les documents de la mairie.

Les acteurs des 07 quartiers disposant d'un puits d'eau (Fiankop, Tchoualé, Famla, Haoussa, Tsinkop, Melang et Siteu) ont été enquêtés, dans deux catégories, riverain de puits et membre d'association de quartier, impliquant pour chaque catégorie 2 hommes, 2 femmes, 2 jeunes, et le chef de quartier, soit 91 personnes. Les acteurs des 06 villages disposant d'un forage (Aza'a, Nzembing, Ndenkwop, Keuli, Fotetsa, Banki) ont aussi été enquêtés, avec la même stratification, 78 personnes. Pour les 9 quartiers disposant chacun d'une source d'eau (Ngui, Tchoualé, Busy Home, Keleng, Atchouazong, Fongo Ndeng, Lefock, Vallée et Fossong Wentcheng), 279 personnes ont été enquêtées, impliquant à chaque fois le chef de quartier, les riverains de la zone de captage, ceux des bornes fontaines et les autres. Le tableau 1 illustre la stratification pour 39 acteurs de l'assainissement du marché des vivres de la ville de Dschang, marché $\mathrm{B}$.

Tableau 1 : Illustration de la typologie des personnes enquêtées, acteurs de l'assainissement du marché B de la ville de Dschang.

\begin{tabular}{|c|c|c|c|c|c|c|}
\hline $\mathbf{N}^{\circ}$ & Structure & Catégorie & Effectif & $\mathbf{H}$ & $\mathbf{F}$ & Total \\
\hline 1 & \multicolumn{2}{|c|}{ Délégué du Ministère du développement Urbain et de l'Habitat } & 01 & $/ /$ & // & 01 \\
\hline 2 & \multicolumn{2}{|c|}{ Délégué du Ministère du commerce } & 01 & $/ /$ & $/ /$ & 01 \\
\hline 3 & \multirow{2}{*}{\multicolumn{2}{|c|}{$\begin{array}{l}\text { Délégué du Ministère des Petites et Moyennes Entreprises } \\
\text { Chef service d'hygiène/chargé du partenariat } \\
\text { Commune de Dschang-AIMF }\end{array}$}} & 01 & // & $/ /$ & 01 \\
\hline \multirow{4}{*}{4} & & & 01 & $/ /$ & $/ /$ & 01 \\
\hline & \multirow[t]{3}{*}{$\begin{array}{l}\text { Commune de } \\
\text { Dschang }\end{array}$} & $\begin{array}{l}\text { Chef de bureau des inspections sanitaires et } \\
\text { domiciliaire chargé de la protection civile }\end{array}$ & 01 & $/ /$ & $/ /$ & 01 \\
\hline & & Vendeurs de tickets de marché & 02 & $/ /$ & // & 02 \\
\hline & & Chauffeur de camion de ramassage & 02 & $/ /$ & $/ /$ & 02 \\
\hline 5 & \multicolumn{2}{|c|}{ L'association des commerçants } & & 02 & 02 & 04 \\
\hline \multirow{3}{*}{6} & \multirow{3}{*}{$\begin{array}{l}\text { Commerçants } \\
\text { fixes }\end{array}$} & Vendant à même le sol & // & 02 & 02 & 04 \\
\hline & & Vendant dans le hangar & // & 04 & 04 & 08 \\
\hline & & Vendant dans les boutiques & $/ /$ & 02 & 02 & 04 \\
\hline 7 & \multicolumn{2}{|c|}{ Commerçants du jour du marché } & & 05 & 05 & 10 \\
\hline \multicolumn{3}{|c|}{ Total } & 09 & 15 & 15 & 39 \\
\hline
\end{tabular}




\section{RESULTATS}

\section{Culture de l'eau et ressenti social dans la Commune de Dschang}

Sur un total de 511 enquêtés, tous trouvent que l'eau est importante que ce soit pour la communauté, l'école ou le marché. L'eau trouvée dans 99,74\% de points (source, puits et forage) sert à boire, à l'hygiène corporelle et santé, et à $99,49 \%$ aux nettoyages divers. Par contre, trois personnes sur quatre $(74,42 \%)$ semblent utiliser l'eau pour l'arrosage de la cours et $69,66 \%$ l'utilise pour l'agriculture (irrigation) ou outil pédagogique.

La Figure 2 indique que l'eau est principalement puisée à la source, dans les puits et forage, rarement dans les réseaux $(30 \%)$.

Au sujet du temps nécessaire pour aller chercher de l'eau, les informateurs présentent dans l'ensemble, une moyenne de $21 \pm 28$ minutes (Figure 3). Beaucoup de temps sont donc nécessaires pour aller puiser de l'eau au point de ravitaillement.

\section{Durabilité incertaine pour des points d'eau aménagés}

Tous les 04 mini réseaux d'eau potable réalisés dans la Commune de Dschang par la société suédoise ScanWater sont non fonctionnels. Des 22 points d'eau aménagés en 2008, seulement $13(59 \%)$ sont fonctionnels. Dans les localités où les points d'eau ne fonctionnent pas, les populations font recours à diverses autres sources d'approvisionnement, à savoir à la rivière, à la source, l'eau des pluies, au puits non aménagé, etc.

\section{Raisons évoquées comme cause de l'échec des projets antérieurs}

Seules $44,39 \%$ de personnes interrogées savent comment sont gérés ces points d'eau. 41,29\% de personnes estiment que le point d'eau fonctionne régulièrement.
Sur le terrain, 06 points d'eau (27\%), notamment les forages n'ont jamais fonctionné. On a aussi les bornes fontaines de certains réseaux gravitaires comme à Ngui qui sont tombés en panne après un fonctionnement de début. Certains forages comme à Siteu n'ont jamais été ouverts, le cadenas du scellé étant toujours au service technique de la Commune. Au niveau de la Commune, on annonce comme raison la formation des usagers qui n'était pas encore faite mais qui est programmée pour les mois à venir. L'ensemble de ces raisons et leur proportion se trouvent dans le Tableau 2.

\section{Idées d'amélioration du système selon les enquêtés}

A la question de savoir comment on peut améliorer, les réponses sont nombreuses et varient d'un point à l'autre. On a parlé de l'aménagement d'autres points dans la localité, l'augmentation des débits, de l'achat des équipements durables (robinets, pompes, manivelle, etc.), de bien positionner le point d'eau par rapport aux habitations, d'autoriser l'accès à tous les riverains, de confier les aménagements aux entreprises compétentes, etc. Certains ont insisté sur la gestion de la distribution de manière à limiter les pertes d'eau, d'autres soulignent l'aménagement des alentours des points d'eau, l'implication des populations à la gestion des ouvrages. On a noté chez quelques gens la sollicitation de l'extension du réseau dans les habitations privées. Ceux qui ont des points d'eau non ouverts réclament plutôt leur ouverture. L'amélioration de la qualité de l'eau a été rarement sollicitée.

\section{Solutions mises en œuvre dans la Commune de Dschang pour améliorer durablement le service AEPA}

C'est dans le cadre de la mise en œuvre des recommandations des études antérieures (Temgoua et al., 2009), que la commune a 
créé en 2010 une Agence Municipale de l'Eau et de l'Energie (AMEE), par décision municipale en conformité à la législation sur la décentralisation. L'AMEE est un établissement public communal doté de la personnalité morale et de l'autonomie financière, sous l'autorité d'un conseil d'administration présidé par le Maire. Son objectif principal est d'accompagner la commune dans la participation citoyenne effective des populations à toutes les étapes du projet eau-assainissement-énergie, leur formation aux petites maintenances des ouvrages et à leur gestion. Cette approche est différente, bien sûr, suivant les milieux (plus ou moins villageois ou urbains) et le type d'équipement $(\mathrm{PMH}$, adduction d'eau liée à une source, hydroélectricité, etc.). Mais elle dépend aussi de l'importance accordée par la commune (maître d'ouvrage) et les équipes chargées de la maîtrise d'œuvre sociale, à prendre en compte les besoins, intérêts et aspirations des différents groupes sociaux, intérêts qui ne sont pas forcément les mêmes suivant ces groupes.

Depuis lors sur le terrain, l'AMEE a mis en place, formé les membres et anime mensuellement les comités des usagers pour les bilans et des solutions aux problèmes, soit un total de 40 comités de gestion installés autour de 14 mini-réseaux et un grand réseau d'eau pour environ 25.000 bénéficiaires, 01 mini barrage hydroélectrique qui alimente en électricité un lycée technique, un centre de santé et environ 500 habitations, et enfin une centaine de toilettes publiques. Elle perçoit $30 \%$ des recettes opérées par le comité des usagers et s'occupe des grandes pannes (plus de 100.000 FCFA, environ $150 €$ ) survenues sur les ouvrages. Pour la pérennité de ces actions, les membres de bureau des comités des usagers sont élus démocratiquement; les comités défaillants sont aussitôt remplacés. Certains comités d'usagers ont mobilisé un approvisionnement financier leur permettant d'entrevoir une extension pour certains, la construction du réservoir pour d'autres.

Les impacts directs de la nouvelle gouvernance décelés sur le terrain dans la Commune de Dschang, portent sur la pérennité du service d'AEPA, la diminution du temps de corvée et, la diminution des maladies hydriques. Les résultats de l'enquête indiquent que plus de $92 \%$ d'usagers admettent que les points d'eau fonctionnent normalement. Selon nos observations, la fraction résiduelle est constituée des familles rurales qui exploitent encore les femmes et les enfants pour la corvée de l'eau et refusent de ce fait de payer la moindre contribution pour obtenir de l'eau. Les recommandations pour améliorer la situation varient d'un point à l'autre. Les populations proposent l'aménagement d'autres points dans la commune, l'augmentation des débits pour certains points existants et l'extension du réseau pour d'autres. Une fraction faible sollicite de baisser le prix de l'eau, voire même annuler la contribution des usagers.

La Commune de Dschang a remporté, au mois de novembre 2016, le deuxième prix national du concours des bonnes pratiques communales, organisé par le Fonds national d'équipement intercommunal (Feicom). La distinction récompense la mise en place de l'Agence municipale de l'eau et de l'énergie (AMEE) et son bon fonctionnement. La commune a reçu pour cela la somme de 20 millions FCFA (environ 30 500€). 


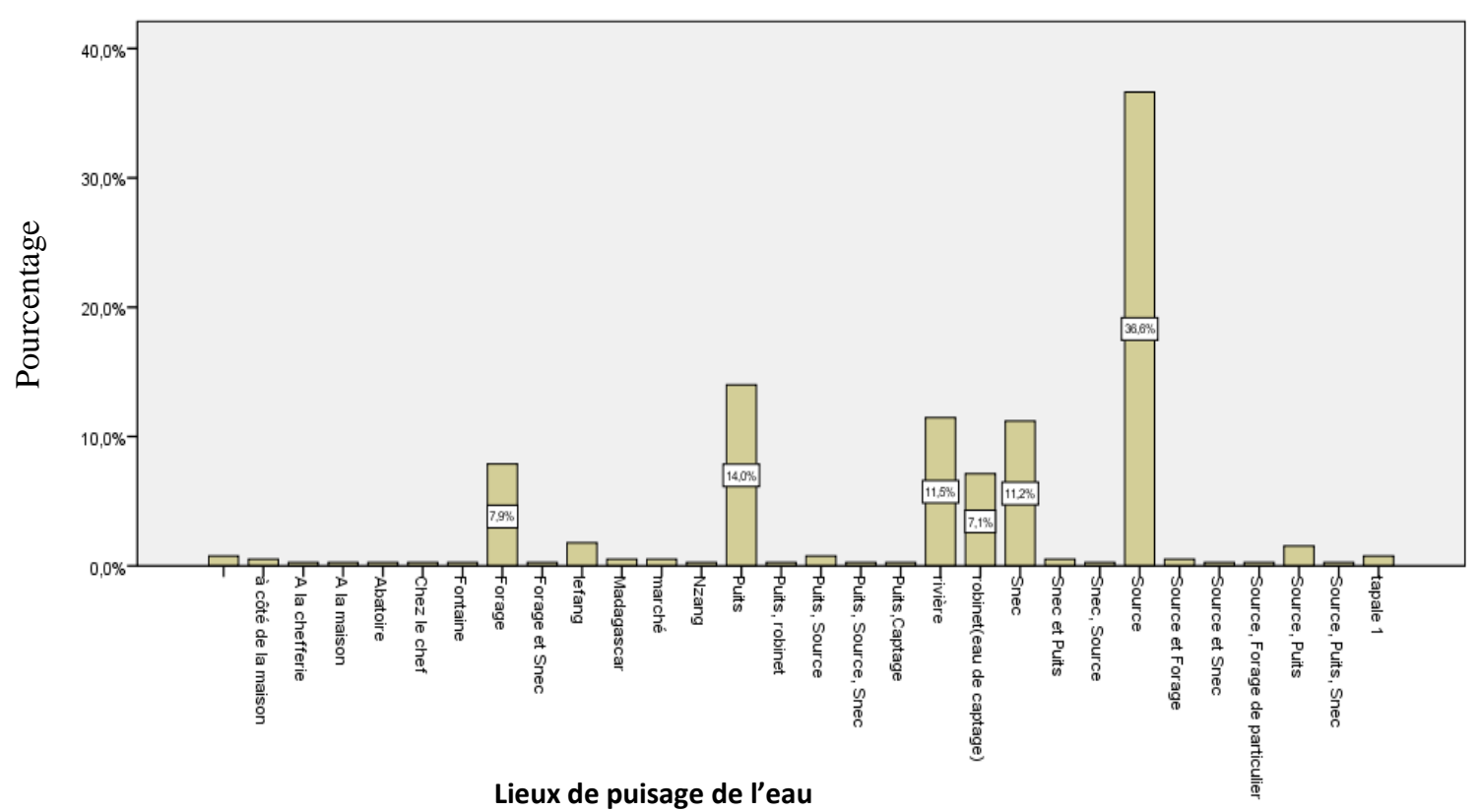

Figure 2 : Différents lieux de puisage de l'eau par les usagers de la Commune de Dschang.

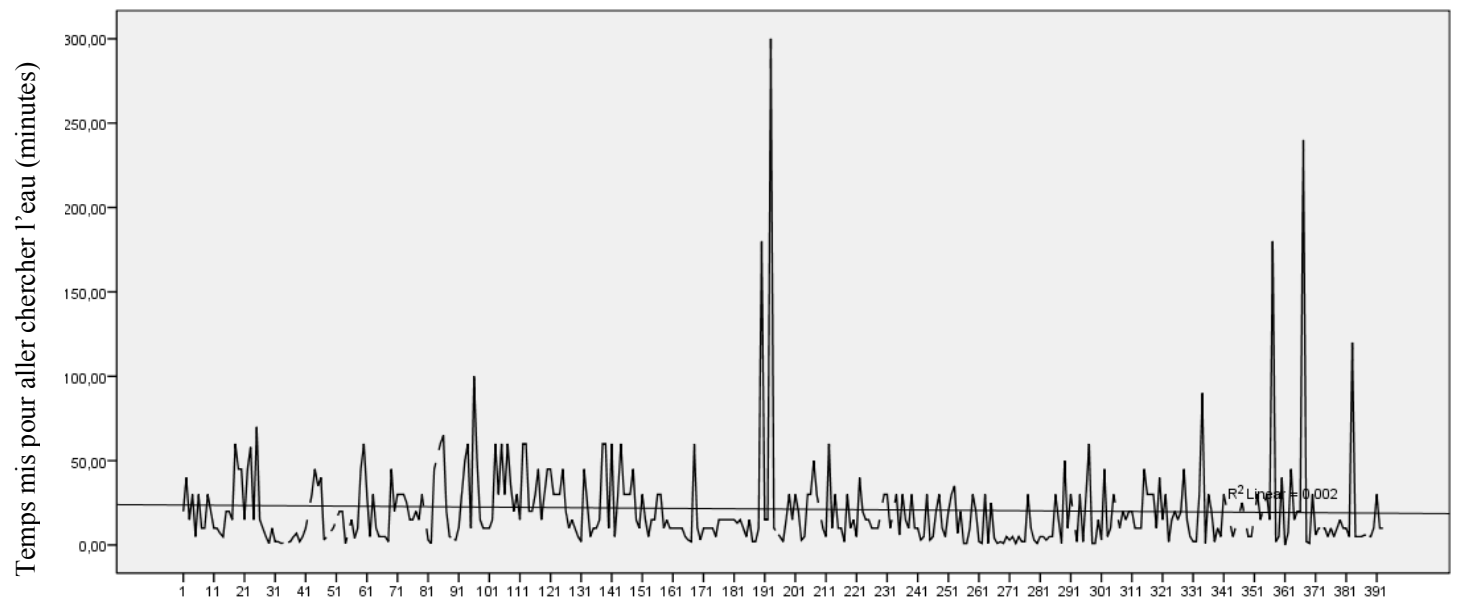

\section{Numéro des répondants}

Figure 3: Temps mis pour aller chercher l'eau pour différents usages dans la Commune de Dschang.

Tableau 2 : Raisons évoquées pour le dysfonctionnement des points d'eau dans la Commune de Dschang.

\begin{tabular}{lcc}
\hline & Nombre & Pourcentage \\
\hline Manque d'entretien / suivi & 170 & 43,3 \\
N'a jamais fonctionné & 126 & 32,1 \\
Panne d'un équipement & 162 & 41,2 \\
C'est toujours fermé & 104 & 26,5 \\
\hline
\end{tabular}




\section{DISCUSSION}

\section{L'accès limité à l'eau n'est pas lié à la disponibilité}

Le présent travail sur le ressenti sur l'eau dans la Commune de Dschang trouve qu'il y a un lien étroit entre la nature de la ressource, la qualité de l'ouvrage hydraulique, le consentement à payer des usagers et les habitudes de ces usagers qu'il faut associer pour trouver comment améliorer la durabilité des services AEPA. L'inaccessibilité de la population à l'eau potable est due à la faible couverture du réseau public d'adduction d'eau, ce qui amène la majorité de la population à consommer les eaux des puits traditionnels dont l'aménagement et l'hygiène sont inacceptables. La situation a été aggravée vers les années 1980 avec l'échec des entreprises publiques qui étaient censées assurer la fourniture du service de l'eau dans la plupart des pays (Tanawa et al., 2002; Temgoua et al., 2009). Même dans les régions où les ressources en eau sont abondantes, la question de l'accès n'est pas toujours résolue. Depuis les années 2000, l'accès à l'eau est au cœur des objectifs du Développement Durable. A l'ouverture du congrès de l'AfWA (2018), son président a fait remarquer que "la ressource en eau est la plus affectée par les Changements climatiques et que face à cette situation caractérisée, 400 millions de personnes sont privées d'eau potable en Afrique et pour l'assainissement, son accès reste un luxe pour 700 millions de personnes". Baron et Bonnessieux (2011) montrent que les tensions qui se manifestent autour des points d'eau ne sont pas seulement liées à la diversité des écosystèmes. Elles sont fonction de la disponibilité des infrastructures hydrauliques, de leur état de fonctionnement et de la nature de leur mode de gestion. La construction de multiples ouvrages hydrauliques ScanWater au Cameroun, l'aménagement de 22 points d'eau dans la Commune de Dschang en 2008 par les partenaires au développement, etc, n'ont pas résolu le problème de l'accès à l'eau dans la localité. D'ailleurs, Lobina et Hall (2008) reconnaissent qu'après cet échec, d'autres modes de gouvernance ont émergé mais le service public reste à privilégier.

\section{Modélisation des solutions entreprises par la Commune de Dschang}

Au vu des échecs de nombreux projets d'AEPA, la commune a commis en 2010 une étude sur le ressenti socioculturel de l'eau. Il peut être conçu, comme Baron et Bonnessieux (2011), qu'au-delà des facteurs environnementaux et des contraintes liées aux modalités d'accès à l'eau, les réalités de terrain font apparaître deux types de facteurs pour interpréter la nature des conflits dans l'accès à l'eau. L'argument fort à présenter à la suite de ce travail est que la prise en compte des contextes socioculturels est une condition incontournable pour que les projets d'eau potable et assainissement remplissent leurs objectifs d'amélioration de la santé et des conditions de vie des populations. D'une part, il existe un décalage entre les représentations et les normes qui sous-tendent l'implantation des points d'eau et leurs modes de gestion. D'autre part, il importe de prendre en considération à la fois la diversification des usages et l'hétérogénéité des usagers de l'eau pour comprendre l'accroissement des disparités au sein des territoires, tant en milieu urbain que rural. $\mathrm{Au}$ niveau des zones d'intervention des projets, le but final est une prise de conscience de la part de la population sur l'importance de l'eau, hygiène et assainissement. On doit inviter les populations, toutes classes et âges confondus, à participer dans un processus qui vise à changer leur comportement pour améliorer la santé de toute la communauté. Et tout ceci en partant d'une optique participative. C'est également au niveau de la zone de projet que les Comités de Gestion sont mobilisés pour convaincre les personnes de contribuer aux 
services d'eau (Jones, 2017). L'approche qu'a adoptée la Commune de Dschang est celle de pourvoir ses services techniques en structure autonome de gestion en régie des services eau et assainissement. Cette structure prend en relaie la mise en place des comités de gestion des ouvrages. Cette mise en place se fait selon les principes démocratiques en laissant les usagers choisir leurs dirigeants. A travers des formations, les membres des comités sont renforcés en éducation financière et accompagnés pour la mise en place de mécanismes qui permettent de financer les infrastructures et les services de maintenance et de réparation des points d'eau. Jones (2017) ajoute que quand une communauté est convaincue des bénéfices de services AEPA et est disponible à payer pour ceux-ci, ses membres deviennent des clients et non des bénéficiaires, ce qui permet que la demande d'amélioration des services AEPA soit stimulée dès la base. C'est alors que d'autres acteurs, comme le secteur privé, peuvent être motivés à répondre à cette demande. L'accompagnement par les formations, l'audit hebdomadaire sont les nœuds à la pérennisation du fonctionnement des ouvrages.

\section{Conclusion}

Cette étude avait pour objectif d'auditer les services d'AEPA dans la Commune de Dschang et de proposer un modèle de pérennisation des ouvrages. Au vue des échecs de nombreux projets d'AEPA, la Commune de Dschang a commis en 2010 une étude sur le ressenti socioculturel de l'eau. Les solutions entreprises par la Commune de Dschang sont suffisamment louables pour être diffusées auprès des petits opérateurs du secteur d'AEPA. Il en ressort les recommandations ci-après :

Pour un projet d'AEPA réussi au niveau des CTD, on doit impliquer les populations bénéficiaires dès l'idée du projet et constituer le comité des usagers,

$\mathrm{Au}$ niveau des CTD, l'agence municipale est mise en place, s'entoure des techniciens qualifiés pour l'appui technique et de gestion des membres du comité,

> La synergie entre comités des usagers, agence municipale et le conseil municipal assure la pérennité des services.

La perspective d'une agence à une échelle plus grande tel le syndicat des communes pour la maîtrise des services d'AEPA pourrait-elle avoir une plus-value ?

\section{CONFLIT D'INTERETS}

Les auteurs déclarent qu'il n'y pas de conflit d'intérêts entre eux.

\section{CONTRIBUTIONS DES AUTEURS}

ET a analysé les données et a rédigé le manuscrit. VMM a coordonné les enquêtes sociologiques. $\mathrm{MM}$ et $\mathrm{BN}$ ont encadré le travail sur le terrain.

\section{REFERENCES}

Adjagodo A, Agassounon Djikpo Tchibozo M, Kelome Ahouangnivo N, Lawani R. 2016. Flux des polluants liés aux activités anthropiques et risques sur les ressources en eau de surface à travers le monde (synthèse bibliographique). Larhyss Journal, 28 : 7-23.

Agassounon Djikpo Tchibozo M, Ayi-Fanou L, Lozes E, Fadonougbo R, Anago GDJ, Agbangla C, Ahanhanzo C. 2012. Impacts sanitaires liés à l'usage des eaux de puits, à l'assainissement et à l'aménagement à Gbôdjê dans l'arrondissement de Godomey au Bénin. Int. J. Biol. Chem. Sci., 6(2): 592602.

Baron C, Bonnassieux A. 2011. Les enjeux de l'accès à l'eau en Afrique de l'Ouest: diversité des modes de gouvernance et conflits d'usages. Mondes en Développement, 156(4): 17-32. DOI:10.3917/med.156.0017. 
Djegbe I, Tamou-Tabe TS, Topanou N, Soglo FM, Paraiso A, Djouaka R, Kelome CK. 2018. Variation saisonnière de la qualité physicochimique et microbiologique des eaux d'irrigation et des légumes du site maraîcher de Bawéra et risques sanitaires associés. Int. J. Biol. Chem. Sci., 12(2): 781-795. DOI: https://dx.doi.org/10.4314/ijbcs.v12i2.13

Dovonou FE, Alassane A, Adjahossou VN, Agbodo B, Djibril R, Mama D. 2017. Impacts de l'assainissement autonome sur la qualité des eaux de puits dans la Commune de SèmèPodji (Sud-Bénin). Int. J. Biol. Chem. Sci., 11(6): 3086-3099. DOI: https://dx.doi.org/10.4314/ijbcs.v11i6.4

INS. 2016. Annuaire statistique du Cameroun. INS. Jones S. 2017. Mobilisation communautaire dans le secteur EHA: comment assurer la participation de tous et toutes? Rapport de la Revue Technique, Consortium WASH RDC, $24 \mathrm{p}$.

Lobina E, Hall D. 2003. Problems with private water concessions: a review of experience, Public Services International Research Unit (PSIRU), School of Computing and Mathematical Sciences, University of Greenwich, Working Paper.
Lobina E, Hall D. 2008. The comparative advantage of the public sector in the development of urban water supply. Progress in Development Studies, 8(1): 85-101.

Olivier de Sardan JP, Elhadji Dagobi A. 2001. La gestion communautaire sert-elle l'intérêt public? Le cas de l'hydraulique villageoise au Niger. Politique Africaine, 80 : 153-158.

Tanawa E, Djeuda Tchapnga HB, Ngnikam E, Temgoua E, Siakeu J. 2002. Habitat and protection of water resources in suburban areas in african cities. Building and Environment, 37(3): 269-275.

Temgoua E, Ngnikam E, Ndongson B. 2009. Drinking water quality: stakes of control and sanitation in the town of Dschang Cameroon. Int. J. Biol. Chem. Sci., 3(3): 441447.

Temgoua E. 2011. Chemical and Bacteriological Analysis of Drinking Water from Alternative Sources in the Dschang Municipality, Cameroon. Journal of Environmental Protection, 2: 620-628. DOI: 10.4236/jep.2011.25071. 